\section{BIOTECNOLOGÍA, SOCIEDAD Y ECONOMÍA: UNA VISIÓN PERSONAL}

\author{
Emilio Muñoz \\ Consejo Superior de Investigaciones Científicas \\ Comité Científico de ASEBIO \\ emilio.munoz@cchs.csic.es
}

\section{BIOTECHNOLOGY, SOCIETY AND ECONOMY: A PERSONAL VIEWPOINT}

Cómo citar este artículo/Citation: Muñoz, E. (2014). "Biotecnología, sociedad y economía: una visión personal". Arbor, 190 (768): a147. doi: http://dx.doi.org/10.3989/ arbor.2014.768n4001

Recibido: 9 junio 2014. Aceptado: 14 julio 2014

RESUMEN: Las relaciones entre biotecnología, sociedad y economía en los contextos europeo y español se han explorado desde una perspectiva evolucionista e integradora a partir de tres espacios: el de las políticas científicas y tecnológicas, el social y el de los impactos socioeconómicos. Se concluye que la(s) biotecnología(s) supone(n) un reto y una esperanza. Un reto por su complejidad, por su riqueza conceptual y por la exigencia de combinar cualidades y recursos. Una esperanza porque estas propiedades permiten plantear estrategias basadas en los conocimientos científicos y técnicos y en los recursos humanos que pueden representar una alternativa de desarrollo industrial al predominio del actual apoyado en lo financiero. Todo ello debe articularse sobre la base de un espacio social que integre la cultura científica, la valoración de la información y la comunicación con la repercusión socioeconómica. Curiosamente, España ocupa una posición favorable para afrontar los retos y para avanzar por la esperanza gracias a la combinación de una serie de factores: políticas científicas inspiradas en un proceso de modernización del país, potencial científico con disposición creativa y emprendedora, junto a la implicación de un conjunto de actores reflejada en la actitud y el desarrollo empresarial con la colaboración público-privada como eje.

PALABRAS CLAVE: Política científica; biotecnología moderna; biotecnología contemporánea; estudios CTS; espacio social; percepción de la ciencia; comunicación de la ciencia; cultura científica; bioeconomía.
Copyright: (c) 2014 CSIC. Este es un artículo de acceso abierto distribuido bajo los términos de la licencia Creative Commons Attribution-Non Commercial (by-nc) Spain 3.0.

ABSTRACT: The relationships between biotechnology, society and economy in the European and Spanish contexts have been explored from an evolutionary and integrated perspective along three dimensions: scientific and technological policies, the social dimension, and in terms of their socio-economic impacts. It has been concluded that biotechnology in its various forms represents challenges and hopes. The challenges arise out of the complexity, conceptual richness and the need to combine qualities and resources underlying biotechnological approaches. These approaches create hopes because it is possible to develop knowledge and human resources based strategies for industrial development that may be an alternative to the current predominant addressing only financial objectives. All these dimensions have to be supported on a social space integrating scientific culture and the validation of scientific information and communication with the socioeconomic impacts. Spain is in a good position from which to face the challenges and to advance with optimism thanks to a combination of factors: sensible scientific policies built around the objective of the modernisation of the country, an entrepreneurial pool of human resources, joined to the involvement of all stakeholders who have been acting with the public-private collaboration as an axis for entrepreneurship.

KEYWORDS: Scientific policies; modern biotechnology; contemporary biotechnology; STS studies; social space; science perception; science communication; scientific culture; bioeconomy. 


\section{DECLARACIÓN INTRODUCTORIA}

La declaración por parte del Parlamento español del 2014 como Año de la biotecnología en nuestro país ha desencadenado una serie de iniciativas orientadas a difundir los desarrollos de la biotecnología entre la sociedad española sin olvidar las relaciones de dependencia y conexión que tales desarrollos tienen con los entornos internacionales.

Como viene siendo habitual en mis análisis de las dos últimas décadas, esta contribución se armará sobre una serie de pilares: el marco evolutivo aplicado a la integración entre los dos programas de investigación en los que trabajo desde 1991: uno sobre "filosofía de la política científica" y otro sobre "filosofía de la biología". Trataré asimismo de hacer mención a algunos actores españoles, protagonistas de una historia con trayectoria de más de medio siglo, aunque como en el propio título se enuncia el análisis se afronta bajo una perspectiva personal.

\section{LA BIOTECNOLOGÍA Y EL ÁMBITO DE LA POLÍTICA CIENTÍFICA}

\section{Carácter evolutivo de la biotecnología}

Es un hecho reconocido dentro del campo que la biotecnología es tan antigua como la humanidad. Por ello se ha promovido una división de la biotecnología según la evolución temporal que distingue en primer lugar a la biotecnología antigua, que se ha orientado fundamentalmente a las aplicaciones destinadas a la conservación y transformación de alimentos pero que se extiende en el área sanitaria al descubrimiento de las vacunas. La biotecnología moderna por su parte se origina a mediados del siglo pasado con los importantes avances en la molecularización de la biología celular: estructura del ADN, código genético, genética molecular y del desarrollo, y marcada de forma especial por los dos descubrimientos seminales para la estrategia de nuevas aplicaciones biotecnológicas como son la ingeniería genética y los anticuerpos monoclonales.

En atención a los impresionantes avances registrados en las últimas décadas en los conocimientos biológicos (las células madre, las ómicas, la biología de sistemas, la biología sintética, la nanobiotecnología, la bioingeniería; entre otros nuevos campos que surgen y evolucionan con rapidez) he propuesto que se debe incorporar el concepto de biotecnología actual o contemporánea (Muñoz, 2008a).

\section{Dimensión internacional de la política científica en} biotecnología

En opinión personal, la introducción de la biotecnología como prioridad en la política científica coincide con la biotecnología moderna. La primera iniciativa de promoción de la biotecnología en política, de nuevo según mi opinión, fue estadounidense: deriva de un presupuesto de ciencia y tecnología evacuado desde la Oficina del Presidente (Casa Blanca) correspondiente (Ciencia+ presupuesto). En ese presupuesto correspondiente a la segunda mitad de la década de 1970, en una relación no muy numerosa de líneas presupuestarias, aparecía una específica dedicada a la Biotecnología y dotada con 500 millones de dólares. Esta decisión política se completaba en la rúbrica destinada a los proyectos orientados o tecnológicos con el detalle de que se promovía que las grandes empresas farmacéuticas y sanitarias deberían actuar en cooperación con los centros de producción de conocimiento biomédico básico con el objetivo de desarrollar nuevos fármacos y diagnósticos en el diseño de estrategias de ataque a problemas de salud relevantes.

En el ámbito europeo, el primer trabajo, una mezcla de prospectiva y diseño en política científica, se encuadra dentro del ejercicio bautizado como FAST, Forecasting and Assessmentin Science and Technology (Commission of the European Communities, 1978, 1984). A partir de ese ejercicio FAST, la biotecnología entró a formar parte de los Programas Marco de Investigación y Desarrollo Tecnológico europeos. Deforma coincidente, la Organización para la Cooperación y el Desarrollo Económico (OCDE) comenzó a trabajar intensamente sobre la biotecnología, promoviendo con la participación de grupos de trabajo con expertos nacionales, estudios sobre esta tecnología que condujeron a la consideración de la biotecnología como área (multisectorial) de incidencia estratégica en el desarrollo económico de los países avanzados, los integrantes de esa organización.

Fruto de estos trabajos ha sido la definición de la biotecnología que, con alguna matización personal, se puede articular del siguiente modo: "La biotecnología persigue la aplicación del potencial de los seres vivos, de su componentes o de sus partes para transformar sustancias con resultado de cambio en alguna(s) de sus propiedades o para generar nuevos productos y /o procesos destinados a conseguir aplicaciones en diversos sectores de la vida económica y social“.

De esta compleja definición, cabe concluir que no hay una sola biotecnología y que por lo tanto hay que hablar de biotecnologías en plural también desde el 
punto de vista de los sectores (tradicionales o renovadores) en que se aplican. Por eso se ha distinguido entre biotecnologías según colores: la biotecnología roja o biotecnología de la salud humana- la salud animal está en litigio entre esta biotecnología roja y la verde-; la biotecnología verde que abarca el sector agroalimentario (alimentos obtenidos por técnicas biotecnológicas, alimentos funcionales, trazabilidad alimentaria, producción agroforestal por técnicas biotecnológicas, y salud animal); la biotecnología blanca que incide sobre la industria en relación con la mejora de procesos y nuevos materiales; la biotecnología azul que recurre a los recursos marinos y en general a los acuáticos; a estas cuatro, como consecuencia de la evolución expansiva y bajo la perspectiva del año de la biotecnología, se han propuesto otras dos: la gris asociada con la protección del medio ambiente por medio de procesos o productos como la biorremediación, los biocombustibles, la biodiversidad, y por último, la naranja que se relaciona con la aplicación cada vez más influyente de la informática y la computación al desarrollo biotecnológico.

Como se procurará hacer evidente en el próximo apartado, España ha conseguido con las biotecnologías algo bastante inaudito en el terreno de la alta tecnología: incorporarse al tren de su desarrollo en fase temprana. Esto permitió que se pudiera actuar en el seno de las organizaciones internacionales, la Europa comunitaria y la OCDE, con voz propia. En esta tarea hay que destacar el papel de actores relevante como Armando Albert y Regina Revilla. El primero en su condición de asesor desde 1983 para los temas biotecnológicos en la Dirección General de Política Científica que asumía la Secretaría General de la Comisión Asesora de Investigación Científica y Técnica (CAICYT)- adscrita a la Secretaría de Estado de Universidades e Investigación del Ministerio de Educación y Ciencia-. Regina Revilla por su parte colaboró muy eficazmente en todas las actividades europeas relacionadas con la biotecnología desde la perspectiva de la empresa y la industria como subdirectora responsable de estos temas en el Centro para el Desarrollo Tecnológico Industrial (CDTI).

\section{La dimensión española de la política científica en biotecnología}

Como se acaba de apuntar, España ha tenido una situación favorable, y bastante única, respecto a la política científica en biotecnología. Nuestro país tuvo la oportunidad de que la génesis de estas incitativas en el contexto internacional coincidiera con el mo- mento de la transición política hacia la democracia en España. Se pudieron por lo tanto aprovechar una serie de hechos positivos previos como: la corta pero importante existencia, iniciada en 1981 y clausurada en 1982, del Ministerio de Universidades e Investigación que tuvo un efecto decisivo en la (re)organización del sistema encargado de la gestión de las políticas de ciencia y tecnología en España.

Factor decisivo para el futuro de la política científica fue que esta política se incorporara en la agenda del Partido Socialista Obrero Español (PSOE) mientras estaba en la oposición, y que formara parte del programa electoral presentado para las elecciones de octubre de 1982 que fue diseñado como un programa de reforma del sistema español de ciencia y tecnología dentro de un proyecto general de la modernización de España.

\section{Algunos hitos de esta política sobre biotecnología en el periodo 1982-1986}

- Lanzamiento del Programa Movilizador de Biotecnología.

- Presentación de candidatura española para la sede del Centro Internacional de Ingeniería Genética y Biotecnología (CIIGB), una iniciativa promovida por la Organización de Naciones Unidas para el Desarrollo Industrial (ONUDI).

- Propuesta de creación del Centro Nacional de Biotecnología, aprovechando la dinámica generada por la iniciativa anterior. El programa de gestión para la creación de este Centro fue plenamente innovador y probablemente pionero en la historia de España introduciendo la fórmula de un Comité Científico Internacional Asesor para seguir y evaluar este proceso.

- Informe de Investigación para el Gabinete de la Presidencia de Gobierno sobre Nuevas Tecnologías, Economía y Sociedad. Este informe fue dirigido por el renombrado sociólogo Manuel Castells y contó con el apoyo del Gabinete de la Presidencia que dirigía Roberto Dorado. Fue publicado por Alianza Editorial (Castells et al., 1986) en dos volúmenes y prologado por Felipe González. En el primer volumen se recogían valiosos análisis sobre las nuevas tecnologías: microelectrónica, informática, telecomunicaciones, automatización, laser, biotecnología, energías renovables, nuevos materiales, así como los sectores industriales productores de nuevas tecnologías en España. En la segunda parte de este primer volumen se abordó el impacto de las nuevas tecnologías en la relación entre España y la Economía Mundial. 
En el volumen dos se exploraban los efectos de las nuevas tecnologías sobre la estructura económicosocial. Este concepto se analizó desde diferentes perspectivas: en su conjunto, sobre las empresas fabricantes de automóviles; sobre el nuevo sector bancario; sobre la agricultura española; asimismo se profundizaba en la relación entre las nuevas tecnologías y la defensa. En otra parte se analizó la relación entre nuevas tecnologías y la vida cotidiana (el hogar electrónico, informática y educación, la informatización de la Administración Pública, las nuevas tecnologías en la medicina, análisis sobre una nueva vida cotidiana). La parte final se ocupaba de analizar los elementos para el desarrollo de un sistema de relaciones entre innovación tecnológica, industria y sociedad en las condiciones específicas de España y que daba el siguiente índice de temas: introducción; el retraso tecnológico español; políticas de desarrollo tecnológico: problemática y orientaciones generales, hacia un sistema Ciencia-Tecnología-Industria; la constitución de un tejido tecnológico industrial, el acompañamiento social e institucional del desarrollo tecnológico de España; los recursos humanos necesarios para la modernización tecnológica de España; la implementación del desarrollo tecnológico.

\section{La puesta de largo de la política científica en biotecnología}

La tarea de modernización del sistema científicotecnológico español alcanzó un punto culminante con la aprobación y promulgación de la Ley de Fomento y Coordinación General de Investigación Científica y Técnica (conocida popularmente como Ley de la Ciencia, ley 13/1986 de 14 de abril). Esta ley se complementó respecto a las cuestiones que nos ocupan con la aprobación y promulgación de la Ley General de Sanidad (ley 14/1986 de 25 de abril) por la que se creaba el Instituto de Salud Carlos III como entidad promotora y gestora de la investigación biomédica y médico clínica.

Paso fundamental fue la posterior elaboración y presentación, con la asistencia del Presidente de Gobierno, Felipe González, del I Plan Nacional de Investigación y Desarrollo Tecnológico, instrumento previsto en la ley de la Ciencia, del cual tuve asimismo la fortuna de asumir tanto su redacción como su puesta en marcha, como primer Secretario General del Plan. En él se consagró la biotecnología como una de las prioridades estratégicas nacionales al integrar en el mismo el Programa Nacional de Biotecnología, dentro del área de Calidad de Vida. Este Programa se consideraba heredero del Programa Movilizador de Biotecnología ya mencionado, cuyos resultados se habían considerados satisfactorios y justificaban el valor científico y la repercusión económica y social de la biotecnología (Comisión Interministerial de Ciencia y Tecnología, 1986. Por ello, en el Programa Nacional se reconocía como prioridad la biotecnología y se aprobó con una aportación del fondo nacional del Plan de I+D para el cuatrienio 1988-1991 de seis mil quinientos cincuenta y cuatro millones de pesetas a los que se sumaba una financiación previa de carácter sectorial por un quinquenio (1987-1991) de algo más de veinte mil trescientos seis millones de pesetas (20.306,4 millones ptas.).

\section{La trayectoria estacionaria de la política científica en biotecnología}

A partir de ese momento álgido, la "moderna" política científica española en la que se había reconocido e integrado la biotecnología como prioridad nacional, entraba teóricamente en estado estacionario, aunque eso no fuera una realidad, experimentando avatares diversos, picos, valles y descensos, a causa de algunos males endémicos de la política científica española y que se pueden resumir en dos: inestabilidades financieras y escasa tradición económica y administrativa en España en la gestión de las políticas de ciencia y tecnología (sobre las patologías de la política científica y las plagas que la afligen véanse Muñoz, 2009, 2014).

Quizá los hechos más significativos en el ámbito público en relación con las políticas de ciencia y tecnología sobre la biotecnología desde aquellos momentos hasta nuestros días sean: el mantenimiento de la biotecnología como prioridad en los Planes Nacionales, el establecimiento de dos centros de investigación biomédica bajo el estatuto de Fundaciones y con financiación público-privada: el Centro Nacional de Investigaciones Oncológicas (CNIO) y el Centro Nacional de Investigaciones Cardiovasculares (CNIC) así como la creación de la Fundación Genoma y la apuesta de Cataluña por la investigación biomédica.

\section{Algunas referencias para pensar}

Como muestra de los análisis realizados por quien escribe sobre estas cuestiones como fruto de la convergencia entre los programas de política científica y biología bajo una aproximación filosófica cabe citar los cinco primeros editoriales de la segunda etapa del Boletín Perspectivas del sector biotecnológico editado online por ASEBIO (Muñoz, 2007). Esta línea ha seguido presente aunque no con esta intensidad ni focalización a lo largo de más de setenta editoriales que se han publicado desde entonces. 


\section{BIOTECNOLOGÍA Y SOCIEDAD}

\section{Estudios CTS (Ciencia, tecnología y sociedad)}

La conexión entre el desarrollo biotecnológico y la sociedad ha sido a la vez consecuencia y causa de las políticas científicas que se han venido delineando.

Esa relación estaba reconocida de forma explícita en el Informe FAST al que se ha hecho mención en el apartado anterior. En las páginas 29-33, en una sección titulada Education: for human resources, and for public understanding se reconoce la relevancia de la educación en las disciplinas científicas y técnicas que son base de la biotecnología y que debe ser pilar para disponer de los recursos estratégicamente más valiosos: los especialistas (expertos) y los innovadores (emprendedores), que desarrollen la(s) nueva(s) biotecnología(s). Este reconocimiento había empezado con la crisis de la década de 1970, momento en que se comienza a cuestionar el modelo de política científica de origen estadounidense y propiciado por Vannevar Bush con su informe al Presidente Roosevelt: Ciencia, la frontera sin límites. Esa situación condujo a una nueva alternativa para comprender la ciencia y la tecnología y sus relaciones con la sociedad. Ese movimiento ha sido conocido como los estudios CTS (en inglés: Science, Technology and Society o también Science and Technology Studies) y tuvo su primera repercusión en los Estados Unidos y en algunos países europeos, nórdicos y del centro. En España, la llegada de este movimiento fue más tardía, apareciendo en la década de 1990. Un libro de referencia para dar a conocer la situación del estado social de la ciencia y la tecnología es el publicado en 1996 (González García, López Cerezo y Luján López, 1996).

\section{Europa y los estudios sobre ciencia y tecnología en perspectiva social}

En Europa existía una clara preocupación por estos temas que pronto cristalizó en las primeras encuestas sobre la percepción social de la ciencia y la tecnología. A esta dinámica iniciada en la década de 1970, se sumaron rápidamente las encuestas sobre cuestiones científicas y tecnológicas sensibles para la ciudadanía como las que ofrecen las biotecnologías y sus conexiones con bienes comunes como la salud y el medio ambiente.

Las encuestas europeas sobre estas cuestiones han sido canalizadas por medio de los Eurobarómetros y en ellos el tema de la biotecnología se empezó a abordar por consorcios europeos.
Una publicación de referencia que recoge una síntesis de estos trabajos, se ofrece en el libro Radiografía de la investigación pública en España (Muñoz et al., 2006). Este libro es resultado de una iniciativa interesante que fue puesta en marcha en 2004 con un importante apoyo institucional por parte del CSIC y sus órganos de dirección, se trataba de la red CTI (Red de Estudios políticos, económicos y sociales sobre la ciencia, la tecnología y la innovación). La Red CTI ha desarrollado a lo largo de ocho años (2004-2011) una intensa actividad (http://www.redcti.csic.es) bajo la acertada dirección del investigador Jesús Sebastián hasta su jubilación que ha coincidido con la crisis y los cambios institucionales, circunstancias que han propiciado el cese de las actividades de la red.

\section{La I+D y la sociedad española}

La obra Radiografía de la investigación pública en España (Sebastián y Muñoz, 2006) dedica su capítulo 6 , con título casi idéntico al de este apartado, a explorar "la compleja red de interacciones de los procesos de generación, difusión y aplicación de los conocimientos y tecnologías con dirección hacia la sociedad como demandante y receptora de los mismos" por medio de tres artículos (Muñoz et al., 2006; González García et al., 2006; Martín et al., 2006). Los dos últimos discuten, respectivamente, la problemática de la participación ciudadana como parte de la democratización de la toma de decisiones sobre ciencia y tecnología y la panorámica general de la situación de la divulgación científica y tecnológica en España. EI primero analiza en una línea holista dentro de los estudios CTS, la creciente implicación social en el ámbito de la I+D, la evolución del papel social de la ciencia y de la tecnología, los planteamientos y resultados de las encuestas sobre la percepción social de la ciencia y las funciones de la comunicación e información sobre el establecimiento de la cultura científica.

Como síntesis de este trabajo, voy a tratar tres temas: la evaluación social y la percepción y comprensión pública de la ciencia y la tecnología; la medida de la percepción pública de la ciencia y la tecnología bajo una perspectiva crítica, y la comunicación e Información para extraer datos sobre el caso de la biotecnología, sobre el que hemos trabajado profusamente desde los primeros años de la década de 1990.

\section{La biotecnología y su espacio social}

Como se apunta en el artículo glosado, la biotecnología es un modelo para afrontar las relaciones entre tecnologías, no siempre fáciles, con las reacciones 
sociales. Sobre sus desarrollos por su carácter rápidamente evolutivo y naturaleza plurisectorial, se ha generado una serie de debates que afectan a un conjunto de cuestiones: regulación, seguridad, estatuto moral de ciertas aplicaciones. Todo ello se enmarca bajo la orientación generada por el concepto de "sociedad del riesgo" (Beck, 1992), sin olvidar como "gobernar los riesgos" (Luján y Echeverría, 2004), atendiendo a las relaciones que en dicha sociedad se establecen entre ciencia y valores.

Medida de la percepción pública. Encuestas sobre la biotecnología

Los análisis y la medida de las percepciones públicas ante la ciencia y la tecnología en general, y respecto a las múltiples dimensiones que rodean a las aplicaciones biotecnológicas no es un asunto senciIlo: se unen para esta dificultad tanto la complejidad del objeto a analizar como la debilidad de las metodologías que se usan basadas esencialmente en encuestas de opinión pública.

Las encuestas europeas respecto a actitudes ante la ciencia y la tecnología se iniciaron, como ya se había comentado, en 1977. La importancia creciente de la biotecnología fue desplazando este tipo de encuestas generales para orientarlas hacia estudios demoscópicos de opinión sobre la biotecnología como parte de los Eurobarómetros desde 1991.

La primera fue realizada en otoño de ese año: el objeto del estudio fue conocer la actitud de los europeos ante los desarrollos científicos en el campo de la biotecnología, aunque trataba asimismo de valorar (medir) el grado de conocimiento de la ciudadanía europea sobre estas cuestiones y las fuentes de información que les parecían más solventes. La segunda encuesta fue llevada a cabo en la primavera de 1993 con la introducción de nuevas preguntas que se orientaron a valorar los conocimientos, en particular tratando de penetrar en los puntos de vista subjetivos sobre las dificultades de las cuestiones. La tercera investigación demoscópica, puesta en práctica en el otoño de 1996, en una Comunidad ya de 15 miembros, recurrió a un cuestionario revisado con nuevas preguntas, manteniendo un número reducido de las preguntas planteadas anteriormente.

El cuarto sondeo, realizado en otoño de 1999, mantuvo el esquema del anterior cuestionario, aunque se añadieron cuatro preguntas relativas a tendencias y dos a tendencia modificadas, amén de una pregunta procedente del acervo de preguntas de la Unidad de Análisis de la Opinión Pública de la Unión europea.
Los cambios se encaminaron a la simplificación o eliminación de términos tendenciosos.

Algunos resultados y consideraciones sobre estas encuestas

En una ponencia presentada en Oviedo en la primavera de 2002 que fue publicada como Documento de Trabajo del Grupo de Ciencia, tecnología y Sociedad Unidad de Políticas Comparadas, CSIC) y que es accesible en http://digital.csic.es(bitstream/10261/1503/i/ dt.0207 se recogen algunas consideraciones que se enuncian a continuación:

i) Los resultados de estas encuestas plantean dificultades para encontrar una lógica en su interpretación.

Los autores del informe del Eurobarómetro 52.1 (publicado en 2000) señalan que es posible identificar tendencias, pero ante la variedad de los cuestionarios como se acaba de describir, se hace muy difícil comparar; incluso el propio concepto de Eurobarómetro sobre biotecnología parece cuestionable.

ii) Los temas que se someten a escrutinio son complejos, abarcan cuestiones tan diversas como: medida de la cultura científica, actitudes que relejan aversiones al riesgo, valores, discriminación entre costes y beneficios, posición frente a la solidaridad. Esto pone en cuestión la solidez y capacidad discriminatoria de los resultados.

Por ello he insistido sobre la consecuencia de realizar estudios más orientados, más focalizados sobre aspectos específicos de la biotecnología y sus aplicaciones.

iii) En el Informe ASEBIO correspondiente al año 2001 elaboré una serie de argumentos que fueron sometidos a debate organizado por la Fundación Grifols en Barcelona el 4 de diciembre de 2001 y como he señalado puede servir para explicar (comprender) la dificultad del tema y que en resumen permite concluir que: "la interpretación de los resultados (de estas encuestas) es una tarea ardua y difícil que no puede sustentarse en una única dirección explicativa. Es razonable considerar que... intervienen una serie de factores, casi todos los que se han venido considerando... como factores explicativos únicos y aislados de contexto. Cabe mencionar elementos cognitivos que influyen tanto en sentido positivo como negativo; trayectorias en la difusión de la información de acuerdo con el desarrollo científico-técnico; intereses tanto particulares como colectivos y generales; valores culturales y sociales". 
iv) El quinto Eurobarómetro cambió la responsabilidad de la coordinación que recayó en George Gaskell, investigador al frente del programa Life Sciences in European Society, y cuyos resultados fueron publicados como informe, titulado Europeans and Biotechnology in 2002 Eurobarómetro 58 , con una segunda edición de 21 de mayo de 2003). Este informe editado por tres miembros del Methodology Institute de la London School of Economics (George Gaskell, Nick Allum y Sally Stores) recogía la firma de una decena de autores, colaboradores en el proyecto y en el que hay que anotar la ausencia de representante español (parece que ello fue consecuencia de un cambio en la empresa demoscópica con la consiguiente retirada de quien había ostentado la dirección de la representación española, que hasta entonces había ostentado el Profesor Rafael Pardo quien pasó a ocupar además la Dirección General de la Fundación BBVA desde donde ha realizado importantes encuestas sobre biotecnología.

En aquellos momentos se insistía para explicar el caso español respecto a la percepción pública de la biotecnología en las condiciones de contexto. Argumento que probablemente tendríamos que revisar en atención al profundo cambio social experimentado.

\section{Comunicación y difusión de la biotecnología}

En España existe ya un notable nivel de trabajos sobre los procesos de información y comunicación de la ciencia y la tecnología como visible fruto de la acción promocional realizada por diversas fuentes de financiación y gestión de la ciencia y la tecnología no solo públicas sino privadas.

Uno de los temas más tratados en este proceso de difusión hacia la sociedad es el de la política científica y para ello ha sido decisivo el papel de la Confederación Española de Sociedades Científicas (COSCE) que además ha generado como dato positivo la asunción de responsabilidades en este ámbito por parte de las distintas sociedades científicas, entre las que me gustaría destacar, tanto por cercanía como por la naturaleza de las actividades emprendidas, a la Sociedad Española de Bioquímica y Biología Molecular (SEBBM).

Como hemos venido señalando, los temas relacionados con las ciencias de la vida y las ciencias biomédicas han gozado de especial predicamento en la última década -no tanto anteriormente como comentaremos después- que se han publicado bajo el rótulo Sociedad mientras que las tecnologías de la información y comunicaciones (TICS) han trasladado su reper- cusión en esta esfera de los medios a las páginas de economía. La misma separación se detecta entre las ciencias y tecnologías de la vida y las TICS en cuanto al grado de especialización juzgar por su intensidad y frecuencia en suplementos o secciones; las primeras son objeto de suplementos o secciones dedicadas a la ciencia y la tecnología mientras que las segundas se encuadran preferentemente en los textos o secciones que se ocupan del desarrollo socioeconómico o en las páginas destinadas a la investigación social.

La contribución publicada en el libro de revisión del sistema público de I+D editado por Sebastián y yo mismo (2006) que recoge una síntesis de las aportaciones realizadas por nuestro grupo a los temas de comunicación y difusión desde 1995 hasta 2005 (Muñoz et al., 2006) termina con un texto, presentado como conferencia en la sede de Valencia de la Universidad Internacional Menéndez Pelayo en un Curso sobre Perspectivas actuales de Filosofía y Metodología de la Ciencia dirigido por el Profesor Wenceslao J. González durante los días 4-8 de julio de 2005 y posteriormente recogido en un libro (Muñoz, 2006).

La lectura puede ser de interés para clarificar estas cuestiones, pues por un lado se hace eco de las mencionadas diferentes trayectorias de las tecnologías de la información y las comunicaciones y las tecnologías relacionadas con las ciencias de la vida y las ciencias biomédicas.

En el trabajo que estamos glosando (Muñoz et al., 2006) se apuntaban dos recomendaciones que estimo siguen vigentes no para atraer atenciones sino para consolidar propuestas y decisiones. Las trascribo a continuación con alguna modificación para actualizarlas:

-Los estudios sobre ciencia y sociedad constituyen un elemento básico para el análisis y el diseño de la políticas científicas y tecnológicas (sin olvidar la innovación ahora en candelero) por lo que deben promoverse tanto en la financiación de programas y proyectos como en el reconocimiento profesional de las tareas educativas e investigadoras.

-Estas propuestas de promoción y reconocimiento deben ser adoptadas por todos los niveles políticos (Europa, España, Comunidades Autónomas) y organizativos (instituciones y actividades docentes e investigadoras).

\section{Impactos socio-económicos y biotecnología. Apuntes} sobre bioeconomía

La preocupación por los impactos económicos de la biotecnología ha estado muy presente desde los momentos en que surge como prioridad en la política cien- 
tífica y que no hace sino intensificarse a medida que esta trayectoria política coincide con los enormes efectos sociales y políticos generados por el uso masivo de las tecnologías de la información y las comunicaciones.

Esta situación no es sorprendente por cuanto estas tecnologías emergen, como se ha estado describiendo, como objetivos a la par que se instala la crisis del petróleo de 1970 y de la que derivan las dudas y primeros cuestionamientos tanto de la política científica moderna inspirada en el Informe de Vannevar Bush, Ciencia: la frontera sin límites como de la organización de la ciencia asentada sobre la sociología de la ciencia que desarrolló y estudió Robert K. Merton. Son los tiempos en que esas críticas en el ámbito de la ciencia, o de la investigación básica, coinciden en el plano de la economía con cambios macro y microeconómicos en muchas economías de América del Norte y Europa que supusieron la decadencia del modelo fordista de la acumulación de capital y la competencia sobre la base de la producción masiva de bienes y servicios como apunta Richard Whitley en su libro La organización intelectual y social de las ciencias $(2000,2012)$.

A pesar de que la expansión y reorganización de las ciencias biológicas y biomédicas ha determinado que la física ya no sea el reflejo de lo científico y de que la biotecnología haya irrumpido con fuerza en el panorama que busca la recuperación de una economía que se basa en los conocimientos científicos y técnicos, los políticos que dirigen la economía desde la visión ultra liberal parecen incapaces de entender lo que significan y pueden significar las biotecnologías para contribuir a un giro esencial en las prácticas y políticas económicas.

No obstante estas carencias y los problemas que conllevan para diseñar y desarrollar estrategias eficientes, hay dos casos que merecen ser traídos a reflexión para ofrecer informaciones y datos que den apoyo a la idea de que la biotecnología en general y la biotecnología moderna y contemporánea en particular, deben ser consideradas como unas de las tecnologías capacitadoras del siglo XXI para contribuir al desarrollo sostenible.

\section{El estudio europeo BIO4EU}

Este estudio fue realizado en el marco de la Estrategia de Lisboa tras la evidencia de su primer fracaso y la consiguiente refocalización en 2005 hacia los objetivos del crecimiento económico y de conseguir más y mejores empleos. El estudio fue solicitado por el Parlamento Europeo y se llevó a cabo en un periodo de tiempo inferior a los dos años, entre el otoño de 2005 y la primavera de 2007, y se centró en determinar el impacto de la biotecnología moderna (no se contemplaba en el ámbito europeo el concepto de biotecnología contemporánea introducido por nosotros en 2008) en tres grandes campos de aplicaciones biotecnológicas: el de la medicina y los cuidados de la salud; el de la producción agrícola y el área agroalimentaria; y el de la producción industrial, la energía y el medio ambiente. Corrió a cargo de investigadores del Centro Común de Investigación (JRC de su siglas en inglés) adscritos al Instituto para Estudios Prospectivos Tecnológicos de Sevilla (ITPS del inglés), asesorados para este proyecto por un comité de expertos del que formé parte, y fue finalmente publicado en 2007 (Zika et al., 2007).

Las conclusiones económicas más significativas resultantes de aquel trabajo según datos de 2002 son:

- La biotecnología moderna, dentro del ámbito de la biotecnología roja, contribuye directamente al $0,04 \%$ del Producto Interior Bruto de la UE. Además contribuye indirectamente al desarrollo de la I+D sobre biofármacos, moléculas farmacológicas de pequeña talla, vacunas y diagnósticos. Todo ello puede aumentar el desarrollo europeo en terapias selectivas como la sustitución de enzimas defectivos, los ensayos genéticos, productos que aumentan su seguridad en los procesos de producción y el uso de anticuerpos monoclonales y los ensayos de diagnóstico cardíaco en los que los Estados Unidos llevan una gran delantera.

- El impacto de la biotecnología moderna en el ámbito de la biotecnología verde representa entre el $13 \%-23 \%$ de sus cifras de negocios y el $0,01 \%-$ $0,02 \%$ del Producto Interior Bruto de la UE. A eso hay que añadir los productos derivados de la biotecnología en este ámbito agrícola y agroalimentario que dan cuenta del $32 \%-38 \%$ de la cifra de negocios y entre el $1,3 \%$ y el $1,55 \%$ del Producto Interior Bruto europeo comunitario. Asimismo hay que tener en cuenta los efectos indirectos que resultan de las aplicaciones de la biotecnología moderna en la medicina veterinaria y en el diagnóstico de las enfermedades de animales domésticos con las consiguientes repercusiones en la seguridad y en el mantenimiento de la confianza de los consumidores.

- La biotecnología moderna en el sector industrial contribuye alrededor del $0,08 \%$ del Producto Interior Bruto, a lo que hay que añadir los efectos indirectos que resultan en las beneficiosas 
influencias que ejerce la aplicación de la biotecnología industrial sobre las mejoras ambientales y las consiguientes positivas implicaciones de carácter económico.

- En conjunto, se puede estimar que la producción y el uso de productos derivados de la moderna biotecnología generan entre el 1,43\% y el 1,69\% del Producto Interior Bruto de la UE, lo que coloca a esta tecnología en el mismo orden de magnitud de sectores socioeconómicos tradicionales como la agricultura $(1,79 \%)$ o la química $(1,97 \%)$. Contribuye igualmente al empleo de forma intangible ya que se refleja en "mejores empleos" y en la salvaguarda de trabajos al fomentar la competitividad. Reduce el uso de recursos y el nivel de emisiones al aumentar la eficiencia productiva. Incide asimismo en mejorar la calidad de vida con reducción de la carga económica y moral que supone la enfermedad y los problemas de envejecimiento en una población tan cargada de años como la europea.

\section{El caso de España. El efecto motor de la Asociación Española de Bioempresas (ASEBIO)}

Al inicio de este apartado, encuentro necesario hacer una referencia, por breve que sea, a que España disponía de beneficios resultantes de la biotecnología antigua como fruto del proceso de reconstrucción del entramado científico-técnico tras la Guerra Civil. El CSIC fue la institución clave al promover y apoyar los Institutos de Fermentaciones Industriales en Madrid y de Tecnología de Alimentos en Valencia. El primero de ellos desempeñando un importante papel en la industria de derivados del vino bajo el liderazgo de Concepción Llaguno y el segundo con una valiosa estrategia de influir en el sector alimentario con la figura de Eduardo Primo Yúfera al frente. No se puede olvidar tampoco el papel del Instituto del Frío en Madrid, aunque la conexión de este con la biotecnología sea mucho más tenue.

Las políticas científicas y tecnológicas que se han ido desgranando en el primer apartado de este artículo aportaron algunos logros en el desarrollo económico de la biotecnología en España. Dos hechos significativos fueron: en primer lugar y desde el prisma interno, el nacimiento de la empresa Ingenasa; en segundo lugar y bajo la perspectiva internacional, la implantación de la empresa Serono en España con el proyecto de instalación de una planta de producción asociada a la biotecnología moderna en Tres Cantos (Madrid). Esta iniciativa fue el feliz resultado de una colaboración público-privada en la que tuvieron un papel destacado, entre otros, Regina Revilla por el CDTI y Francisco Rubio con el decidido apoyo del propietario F. Bertarelli por la empresa suiza.

Pero ha sido la creación de ASEBIO en diciembre de 1999 el factor decisivo, en mi opinión, para la impulsión del (multi)sector biotecnológico en España. Hay un hecho que me parece importante resaltar por no ser muy frecuente en nuestro país: la insistencia en recordar y conmemorar tal efemérides y los hitos que la acompañaron. Ya se hizo con motivo del décimo aniversario y en 2014 con el cumplimiento del decimoquinto aniversario se ha seguido insistiendo en esta saludable práctica. Quizá ello sea debido, de nuevo en mi opinión muy personal, a que ASEBIO es una organización empresarial atípica en España: es joven; partió de una colaboración público-privada con intervención decisiva del Ministerio de Industria de la época (gracias a las intervenciones de los subdirectores generales Juan Carlos Mampaso y María Luisa Poncela); quiso asumir desde el principio no solo el papel gestor de las empresas establecidas sino el de promotor y el de incubadora en lo posible de nuevas empresas; también desde el inicio procuró mantener la llama científica dentro de la asociación; persiguió llevar a cabo una importante política de comunicación y difusión que ha mantenido en una positiva trayectoria.

Si se me permite continuar con la reflexión personal, pienso que entre los que han contribuido a la génesis y al desarrollo de ASEBIO existe la convicción de que se ha asistido a un "milagro" laico que gustaría compartir con la ciudadanía española, sobre todo porque tal "milagro" ha sido reconocido internacionalmente por instituciones como la OCDE (en sus estadísticas y en sus análisis) y Europabio (la Asociación Europea de Bioempresas).

La política informativa de ASEBIO es un acierto en términos generales y por medio de los Informes anuales, un referente nacional e internacional, y la página web (www.asebio.com) en continua evolución, se suministra información contrastada y relevante tanto para el mundo experto, empresarios, inversores, científicos y autoridades políticas, como también para la ciudadanía inexperta pero curiosa y sobre todo preocupada por el futuro del país.

A continuación unos muy pocos datos para sustentar la idea de que se vive un "milagro" en el entorno español. En plena crisis, el Instituto Nacional de Estadística (INE) presentaba en diciembre de 2013 los da- 
tos correspondientes al año 2012. Esos datos no son buenos en términos absolutos pero sorprendentes si se comparan con la situación general de la $1+D+i$ : muestran un descenso, pero con porcentajes bajos ante la importante reducción experimentada por la inversión en ciencia y tecnología. La disminución en la inversión en I+D interna fue del 3,3\% respecto a 2011, pero todavía se mantenía un nivel razonable de los tres sectores de ejecución, con la Administración Pública contribuyendo al 40,10\%, las empresas el $36,0 \%$ y la Enseñanza Superior el 23,6\%. El número de empresas, a pesar de la volatilidad que ha sido impronta de la biotecnología moderna a lo largo de su reciente historia e incluso en épocas de bonanza económica, registró en el año álgido de la crisis un descenso del 0,5\%. Respecto al capital humano, el número de personas dedicadas a actividades de I+D internas en equivalencia a jornada completa alcanzó las 24.121.3 personas con una disminución del 0,7\% en 2012 ; esta cifra supone el $11,6 \%$ del personal total ocupado en las actividades de I+D y el 1,4 por mil de la población ocupada.

Otro dato digno de ser destacado es que el Consejo Español para la Competitividad, grupo formado por algunos de los empresarios más representativos de nuestro país, destacaba al sector de la biotecnología como uno de los siete considerados claves en su Informe: España, un país de oportunidades, presentado en junio de 2013. La característica más destacada de la biotecnología en el citado Informe es su dinamismo en los últimos años sobre todo desde 2006. Este dinamismo se cifra en la duplicación del número de empresas biotecnológicas con un aumento del 240\% entre 2006 y 2011 , mientras que el empleo aumentaba en un $98 \%$.

\section{La bioeconomía en el horizonte}

Para terminar, unas notas sobre bioeconomía, un término que se ha convertido en nuevo mantra y en aparente motor de la Agenda europea 2020 y como parte fundamental del programa Horizonte 2020, que integra I+D e innovación.

Independientemente de las expectativas que pueda evocar, este término es difuso, hay que reconocer que no es que sea polisémico con variados significados, sino más bien proteico, es decir mantiene un solo significado- la relación entre lo biológico y la economía, pero se aplica a diferentes hechos, tendencias, objetivos de la economía. En un principio se aplicó a la economía biológica en línea con la propuesta inicial de Georgescu-Roegen (1971) y con alguna variante se fue extendiendo su uso a los economistas que se han preocupado por la economía de los recursos naturales, por el medio ambiente y su sostenibilidad. Recientemente, este término ha sido asumido por los economistas que lideran la política económica en la Unión Europea, con lo que han trastocado su orientación y su destino al tratar en esta nueva visión de optimizar al máximo el potencial de los recursos biológicos, de explotarlos buscando la eficiencia con el apoyo de las tecnologías que constituyen base y armazón de las biotecnologías como la biología de sistemas, la biología sintética, las ómicas.

Conviene señalar esta dualidad de interpretación y de uso antes de abrazar ciegamente una línea de desarrollo que, como mínimo, ofrece vericuetos y diversificación, riesgos y beneficios, que en suma reclama planteamientos y análisis éticos: es decir, la necesidad de aplicar interéticas (Muñoz, 2008b).

\section{AGRADECIMIENTOS}

Este trabajo ha contado con el apoyo del proyecto: EPISTEMOLOGÍA Y EXPERIMENTACIÓN: biología y circulacion de prácticas entre la agricultura, la botánica y la biomedicina; FFI2012-34076; financiado por el Ministerio de Economía y Competitividad. 
Beck, U. (1992). The Risk Society. Towards a New Modernity. London: Sage Publications.

Castells, M., Barrera, A., Casal, P., Castaño, C., Escario, P., Melero, J. y Nadal, J. (1986). Nuevas Tecnologías, Economía y Sociedad en España. Madrid: Alianza Editorial.

Comisión Interministerial de Ciencia y Tecnología (1986). Plan Nacional de Investigación Científica y Desarrollo Tecnológico. Madrid: Ministerio de Educación y Ciencia.

Commission of the European Communities (1978). Programa FAST. DirectorateGeneral for Science, Research and Development (DG XII).

Commission of the European Communities in association with the Journal Futures (1984). Eurofutures: the challenges of innovation (The FAST Report). London: Butterworths.

Georgescu-Roegen, N. (1971). The Entropy Law and the Economic Process, Cambridge: Harvard University Press. http://dx.doi.org/10.4159/harvard .9780674281653

González García, M. I., López Cerezo, J. A y Luján López, J. L. (1996). Ciencia, Tecnología y Sociedad. Una introducción al estudio social de la ciencia y la tecnología. Madrid: Tecnos.

González García, M., Todt, O., González Gutiérrez, I., López Cerezo, J. A., Estévez, B. y Luján López, J. L. (2006). Participación pública en ciencia y tecnología. En Sebastián, J. y Muñoz, E. (eds.), Radiografía de la investigación pública en España. Madrid: Biblioteca Nueva, pp. 457-488. http://dx.doi. org/10.1080/11358120609487692

Luján, J. L. y Echeverría, J. (eds.) (2004). Gobernar los riesgos. Ciencia y valores en la sociedad del riesgo. Madrid: Biblioteca Nueva, Organización de Estados Iberoamericanos.

Martín, M. J., Rey, J., Garzón, B., Tigeras, P. y Pérez del Val, J. (2006). Estrategias de divulgación de la ciencia y la tecnología en España desde el sector público. En Sebastián, J. y Muñoz, E. (eds.), Radiografía de la investigación pública en España. Madrid: Biblioteca Nueva, pp. 489-508.

Muñoz, E. (2002). La cultura científica, la percepción pública y el caso de la biotecnología [en línea]. Grupo de Ciencia, Tecnología y Sociedad, CSIC. Documento de Trabajo 02-07 [fecha de consulta: 30 de marzo de 2014]. Disponible en: http://digital.csic.es/bitstream/10261/1503/1/dt-0207.pdf

Muñoz, E. (2006). Conflict between Knowledge and Perception: New Spaces for the Comprehension and Management of the Science around the "New Biology". En González, W. J. y Alcolea, J. (eds.), Contemporary Perspectives in Philosophy and Methodology of Science. A Coruña: Netbiblo, pp. 149-164.

Muñoz, E. (2007). Editoriales [en línea]. Perspectivas del sector biotecnológico español. Boletín de noticias de ASEBIO no 1-6 [fecha de consulta: abril 2014]. Disponible en: www.asebio.com

Muñoz, E. (2008a): Más allá de la biotecnología moderna. Hablamos de biotecnología contemporánea [en línea]. Perspectivas del sector biotecnológico español. Boletín de noticias de ASEBIO, 2a época, $\mathrm{n}$ - 11 [fecha de consulta: abril 2014]. Disponible en: www.asebio.com

Muñoz, E. (2008b). Dinámica y dimensiones de la ética en la investigación científica y técnica. En Ausín, T. y López de la Vieja,
M. T. (coords.), Ética de la investigación, Arbor, CLXXXIV, 730, pp. 197-206.

Muñoz, E. (2009). La crisis de la política científica: patologías degenerativas y terapias regenerativas. A modo de epílogo. En Fernández Esquinas, M. (coord.), Perspectivas teóricas sobre ciencia, tecnología e innovación, Arbor, CLXXXV, 738, pp. 837-850. http:// dx.doi.org/10.3989/arbor.2009.i738

Muñoz, E. (2014). Las siete plagas de la política científica española [en línea]. $M a-$ teria [fecha de consulta: 16 de abril de 2014]. Disponible en: http://esmateria. com/2014/01/19/las-siete-plagas-dela-politica-cientifica-espanola/

Muñoz, E., Plaza, M., Santos, D., Espinosa de los Monteros, J. y Ponce, G. (2006). El espacio social de la ciencia y la tecnología: percepción, comunicación y difusión. En Sebastián, J. y Muñoz, E. (eds.), Radiografía de la investigación pública en España. Madrid: Biblioteca Nueva, pp. 409-456.

Sebastián, J. y Muñoz, E. (2006). Radiografía de la investigación pública en España. Madrid: Biblioteca Nueva.

Whitley, R. (2000). The intellectual and social organization of the sciences $\left(2^{\text {nd }}\right.$. edition). Oxford: Oxford University Press.

Whitley, R. (2012). La organización intelectual y social de las ciencias. Buenos Aires: Universidad Nacional de Quilmes.

Zika, E., Papatryfon, I., Wolf, O., Gómez-Barbero, M., Stein, A. J. y Bock, A.-K. (2007). Consequences, Opportunities and Challenges of Modern Biotechnology for Europe, Joint Research Centre-Institute for Prospective Technological Studies. Luxembourg: Office for Official Publications of the European Communities. 